Article

\title{
A Simple and High-Throughput Analysis of Amatoxins and Phallotoxins in Human Plasma, Serum and Urine Using UPLC-MS/MS Combined with PRiME HLB $\mu$ Elution Platform
}

\author{
Shuo Zhang ${ }^{1,2}$, Yunfeng Zhao ${ }^{2}$, Haijiao Li ${ }^{1}$, Shuang Zhou ${ }^{2}$, Dawei Chen ${ }^{2}$, Yizhe Zhang ${ }^{1}$, \\ Qunmei Yao ${ }^{3}$ and Chengye Sun ${ }^{1, *}$ \\ 1 National Institute of Occupational Health and Poison Control, \\ Chinese Center for Disease Control and Prevention, Beijing 100050, China; \\ zhangshuo6789@sina.com (S.Z.); lihaijiao715@126.com (H.L.); zyz97@263.net (Y.Z.) \\ 2 China National Center for Food Safety Risk Assessment, Key Laboratory of Food Safety Risk Assessment, \\ Ministry of Health, Beijing 100021, China; zhaoyf@cfsa.net.cn (Y.Z.); zhoush@cfsa.net.cn (S.Z.); \\ chendw@cfsa.net.cn (D.C.) \\ 3 The People's Hospital of Chuxiong Yi Autonomous Prefecture, Chuxiong 675000, China; \\ 18987838279@163.com \\ * Correspondence: suncy@chinacdc.cn; Tel.: +86-10-83132660; Fax: +86-10-83132046
}

Academic Editor: Jack Ho Wong

Received: 16 February 2016; Accepted: 20 April 2016; Published: 4 May 2016

\begin{abstract}
Amatoxins and phallotoxins are toxic cyclopeptides found in the genus Amanita and are among the predominant causes of fatal food poisoning in China. In the treatment of Amanita mushroom poisoning, an early and definite diagnosis is necessary for a successful outcome, which has prompted the development of protocols for the fast and confirmatory determination of amatoxins and phallotoxins in human biological fluids. For this purpose, a simple, rapid and sensitive multiresidue UPLC-MS/MS method for the simultaneous determination of $\alpha$-amanitin, $\beta$-amanitin, $\gamma$-amanitin, phalloidin (PHD) and phallacidin (PCD) in human plasma, serum and urine was developed and validated. The diluted plasma, serum and urine samples were directly purified with a novel PRiME technique on a 96-well $\mu$ Elution plate platform, which allowed high-throughput sample processing and low reagent consumption. After purification, a UPLC-MS/MS analysis was performed using positive electrospray ionization (ESI+) in multiple reaction monitoring (MRM) mode. This method fulfilled the requirements of a validation test, with good results for the limit of detection (LOD), lower limit of quantification (LLOQ), accuracy, intra- and inter-assay precision, recovery and matrix effects. All of the analytes were confirmed and quantified in authentic plasma, serum and urine samples obtained from cases of poisoning using this method. Using the PRiME $\mu$ Elution technique for quantification reduces labor and time costs and represents a suitable method for routine toxicological and clinical emergency analysis.
\end{abstract}

Keywords: poisonous mushroom; amatoxin; phallotoxin; PRiME HLB; $\mu$ Elution; LC-MS/MS; biological fluids

\section{Introduction}

Collecting wild mushrooms for food and commercial trade is a traditional hobby in China. However, many edible mushrooms are easily confused with species that are fatally poisonous, which can lead to severe food poisoning [1]. Mushroom poisoning is the main cause of death by food poisoning in China, according to data from the National Management Information System of Public Health Emergencies [2]. Among these toxic mushrooms, the genus Amanita is responsible for the most 
fatalities due to its lethal toxins [1,2], most notably the amatoxins and the phallotoxins, two bicyclic peptide families found in a number of mushrooms [3]. The genera Galerina, Lepiota, and Conocybe are also known to contain amatoxins [4]. Amatoxins, especially $\alpha$ - and $\beta$-amanitin, notorious for their high toxicity, interfere with DNA transcription by binding to and inhibiting eukaryotic RNA polymerase II in hepatocytes. Suppressed mRNA synthesis results in cellular necrosis through the inhibition of protein synthesis, and consequently hepatic failure and renal damage can develop, which may lead to death [5]. Although phallotoxins are not considered to have high causative toxicity, it is still necessary to analyze phallotoxins to help identify the mushroom species [6,7].

The initial symptoms of amatoxin poisoning, such as vomiting, abdominal pain, and watery diarrhea, are very difficult to differentiate from the bacterial gastrointestinal disorder and poisoning caused by other non-amanitin-producing mushrooms, such as Russula, Paxillus and Boletus. After the bacterial infection-like period, these symptoms appear to be diminished (12-24 h), but delayed hepatic and renal dysfunctions then occur $(24-72 \mathrm{~h}$ ) [5,8]. Amatoxins are not bound to plasma proteins, and are mostly eliminated through urine. After ingestion [9], they are detectable for approximately $30 \mathrm{~h}$ in plasma or serum, and up to $72 \mathrm{~h}$ in urine [10]. Therefore, a rapid and confirmatory analysis of amatoxins and phallotoxins in biological fluids is essential for the early diagnosis of mushroom poisoning to avoid confusion with bacterial gastroenteritis or invasive and extensive therapies, including organ transplantation in severe cases.

Although several methods have been described to determine amatoxins and/or phallotoxins in biological fluids, including radioimmunoassay (RIA) [11-13], enzyme-linked immunosorbent assay (ELISA) [14,15], capillary zone electrophoresis (CZE) [16,17], and liquid chromatography (LC) combined with ultraviolet (UV) $[7,18,19]$ or electrochemical detection (ECD) [20-22], various problems, such as false positives, unstable reproducibility, poor confirmatory ability and laborious procedures, have limited their application in practice $[19,23]$. Methods combining LC with mass spectrometry (MS), such as triple quadrupole tandem MS (MS/MS) [24-28], time-of-flight (TOF) MS [29] and matrix-assisted laser desorption ionization time-of-flight MS (MALDI-TOF MS) [30], have been reported for the detection of amatoxins and phallotoxins. LC-MS/MS is a powerful technique allowing high sensitivity, reproducibility and specificity, and is less expensive than high-resolution MS. Therefore, we developed a rapid, convenient high-throughput method for early diagnosis to determine the toxins qualitatively and quantitatively and to avoid internal standards (some of which are commercially unavailable) and expensive high-resolution instrumentation.

Although solid-phase extraction (SPE) is a well-known technique for the effective purification of the complex matrix, most of the available SPE methods require laborious conditioning, evaporation, and reconstitution steps. For blood samples, especially plasma, extra protein precipitation before the clean-up step is necessary. To overcome these drawbacks of the traditional SPE method, the OASIS PRiME HLB $\mu$ Elution 96-well plate (Waters, Milford, MA, USA), a novel micro-SPE platform, was introduced to minimize the time and reagent costs. In this 96 -well plate only $2 \mathrm{mg}$ of sorbents are loaded in each well, thus substantially reducing the amount of sample, solvent and generated waste during the procedure. We took advantage of its excellent ability to remove phospholipids from biological samples to reduce matrix effects. The present study is a validation of this method for the quantification of $\alpha$-amanitin, $\beta$-amanitin, $\gamma$-amanitin, phalloidin (PHD) and phallacidin (PCD) in human plasma, serum and urine using LC-MS/MS. It is also the first study to use the PRiME HLB $\mu$ Elution platform for sample preparation in amatoxin and phallotoxin analysis, which could become the most practical method for routine toxicological and clinical purposes.

\section{Results and Discussion}

\subsection{Optimization of LC-MS/MS}

Mass spectrometric parameters were initially optimized by full scan and daughter scan under positive and negative modes for each analyte using infusion combined with $\mathrm{LC}$. The $[\mathrm{M}+\mathrm{H}]^{+}$ion was 
chosen as the precursor ion for all analytes. Table 1 lists the characteristic ions and collision energy for each compound during multiple reaction monitoring (MRM) acquisition. The proposed structures of the corresponding product ions are presented in the Supplementary Materials section (Figure S1).

Table 1. MRM parameters and retention times for target compounds.

\begin{tabular}{|c|c|c|c|c|c|}
\hline Compound & $\begin{array}{c}\text { MRM } \\
\text { Transition }\end{array}$ & $\begin{array}{c}\text { Cone } \\
\text { Voltage (V) }\end{array}$ & $\begin{array}{c}\text { Collision } \\
\text { Energy }(\mathrm{eV})\end{array}$ & $\begin{array}{c}\text { Retention } \\
\text { Times (min) }\end{array}$ & $\begin{array}{c}\text { Ion Abundant Ratio } \\
\text { (\% of Base Peak) }\end{array}$ \\
\hline$\alpha$-amanitin & $\begin{array}{l}919.5>86.0^{1,2} \\
919.5>259.1^{1}\end{array}$ & 20 & $\begin{array}{l}68 \\
42\end{array}$ & $4.72 \pm 0.2$ & $38 \pm 10$ \\
\hline$\beta$-amanitin & $\begin{array}{l}920.5>86.0^{1,2} \\
920.5>259.1^{1}\end{array}$ & 20 & $\begin{array}{l}71 \\
42\end{array}$ & $4.96 \pm 0.2$ & $47 \pm 9$ \\
\hline$\gamma$-amanitin & $\begin{array}{l}903.0>86.0^{1,2} \\
903.0>243.1^{1}\end{array}$ & 20 & $\begin{array}{l}70 \\
41\end{array}$ & $5.45 \pm 0.2$ & $43 \pm 8$ \\
\hline PHD & $\begin{array}{c}789.4>157.0^{1,2} \\
789.4>86.0^{1}\end{array}$ & 20 & $\begin{array}{l}61 \\
70\end{array}$ & $7.31 \pm 0.2$ & $91 \pm 4$ \\
\hline PCD & $\begin{array}{c}847.0>157.0^{1,2} \\
847.0>86.0^{1}\end{array}$ & 20 & $\begin{array}{l}64 \\
70\end{array}$ & $7.87 \pm 0.2$ & $83 \pm 5$ \\
\hline
\end{tabular}

${ }^{1}$ the confirmation ion transitions; ${ }^{2}$ the quantification ion transitions.

For liquid chromatographic separation, several UPLC columns were tested, including ACQUITY UPLC HSS T3 $(2.1 \mathrm{~mm} \times 100 \mathrm{~mm}, 1.8 \mu \mathrm{m})$, ACQUITY UPLC BEH C18 $(2.1 \mathrm{~mm} \times 100 \mathrm{~mm}, 1.7 \mu \mathrm{m})$ and CORTECS UPLC C18+ $(2.1 \mathrm{~mm} \times 100 \mathrm{~mm}, 1.6 \mu \mathrm{m})$ separation columns under their optimal elution conditions. Since $\alpha$-amanitin $(m / z 919.5>86.0)$ and $\beta$-amanitin $(m / z 920.5>86.0)$ both have high carbon contents and their molecular weights differ by only $1 \mathrm{Da}$, with a ${ }^{13} \mathrm{C}$ abundance of $42 \%-43 \%$, the isotopic substituted $\alpha$-amanitin $(m / z 920.5>86.0)$ could interfere with the detection of $\beta$-amanitin $(m / z 920.5>86.0)$. Therefore, the analytes must be completely separated in LC to avoid interference from each other. The CORTECS UPLC C18+ column resulted in an increase in both the retention and resolution of $\alpha$ - and $\beta$-amanitin, with sharper peak shapes and stronger MS responses.

\subsection{Sample Preparation}

Acetonitrile and methanol were used as common extractants, but the extraction efficiency proved to be unacceptably low in all types of matrices (recoveries were below 50\%). The peptide analytes may have co-precipitated with proteins from the matrix. Moreover, the solvent had to be changed to fit the initial polarity of the following SPE purification. Thus, direct sample dilution is preferred over protein precipitation before the purification step.

SPE is an important technique for biological sample purification. Several SPE cartridges, principally based on two different mechanisms of retention, have been applied to amatoxin analysis [19,23,25-27]. To find the most suitable purification mechanism for all analytes and recovery differences, four types of SPE cartridge were screened in the present study. Based on the ion exchange mechanism, considering the amphoterism of the peptide analytes, a weak anion exchange SPE cartridge (Oasis WAX, $30 \mathrm{mg}, 1 \mathrm{cc}$ ) and a weak cation exchange SPE cartridge (Oasis WCX, $30 \mathrm{mg}, 1 \mathrm{cc}$ ) were tested. For the reversed phase mechanism, HLB and PRiME HLB cartridges were tested. The detailed extraction procedures for WAX, WCX, HLB and PRiME HLB cartridges are shown in the Supplementary Materials. The extraction efficiency was determined using a $10 \mathrm{ng} / \mathrm{mL}$ aqueous standard mixture (Figure 1). For WAX and WCX, satisfactory recoveries could not be obtained for all analytes. Although all these compounds are characterized as bicyclopeptides containing an unnatural tryptophan residue, they bear different substituent groups [31]. The presence or absence of $\mathrm{OH}_{1} \mathrm{NH}_{2}$, $\mathrm{CH}_{3}$, and $\mathrm{COOH}$ makes their acidic-basic properties diverse, so their separation behaviors can be quite different on an ion exchange mode SPE cartridge. HLB and PRiME HLB cartridges showed good recoveries $(75.3 \%-94.2 \%)$ for all analytes. To seek an easier and faster method for routine analysis 
for a large number of samples, we applied a method using a 96-well $\mu$ Elution plate. Compared with common procedures, this preparation method substantially reduces the amount of sample, solvent and generated waste during the procedure, due to the high concentrating ability of the sorbent particles. Additionally, the eluent in the wells was directly diluted for injection in our method, which avoids the time-consuming steps of solvent evaporation or solvent exchange under $\mathrm{N}_{2}$ in other methods.

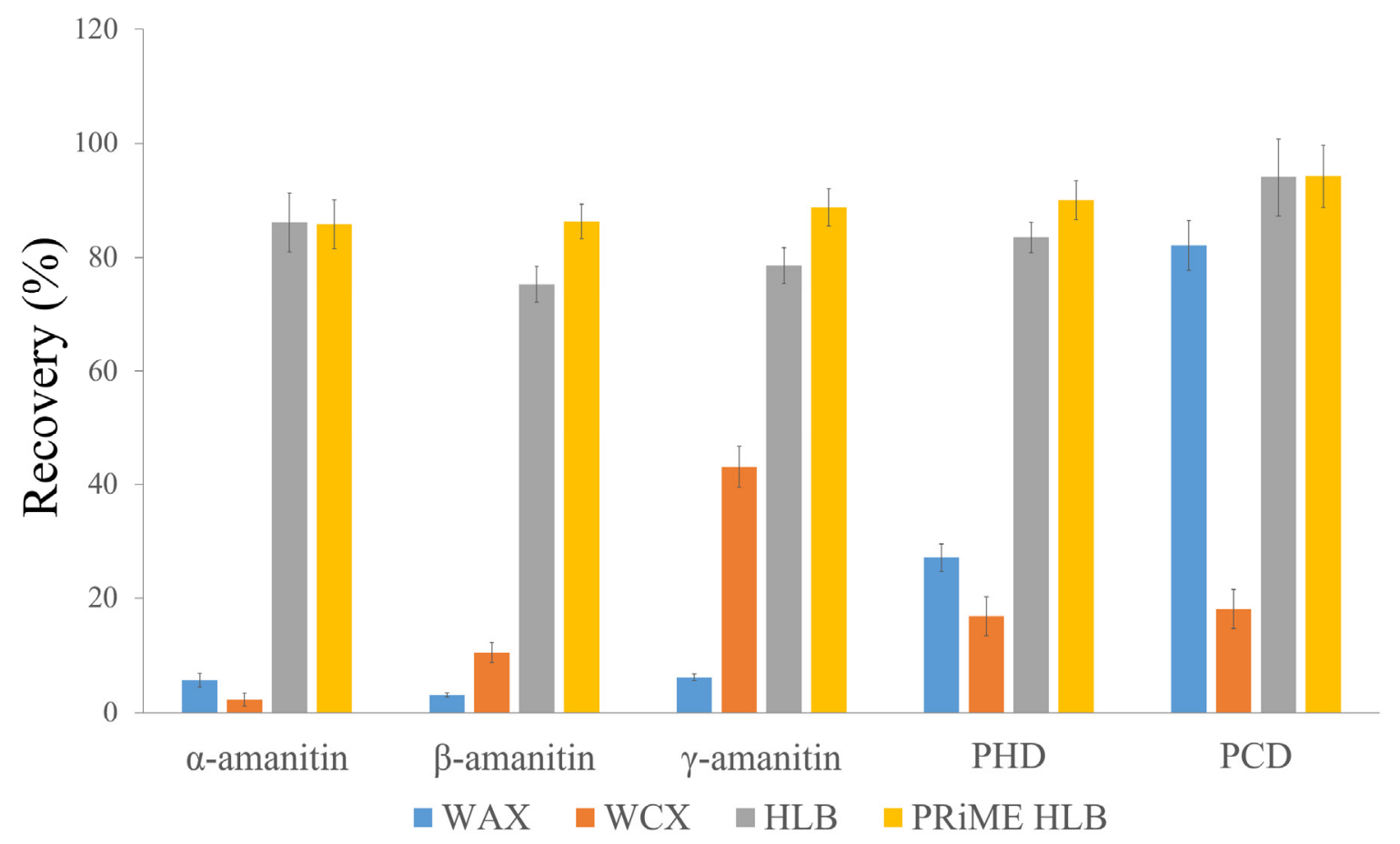

Figure 1. Effects of the different sorbents on the extraction efficiency (recoveries \%) $(n=3)$ of $10 \mathrm{ng} / \mathrm{mL}$ $\alpha$-amanitin, $\beta$-amanitin, $\gamma$-amanitin, PHD and PCD in methanol-water-dissolved standard mixture.

HLB and PRiME HLB mechanisms were chosen for further optimization on the $\mu$ Elution platform. To compare the performance of the two $\mu$ Elution extraction methods, blank samples spiked with $10 \mathrm{ng} / \mathrm{mL}$ plasma were prepared. The HLB $\mu$ Elution and PRiME HLB $\mu$ Elution protocols are shown in the Supplementary Materials. Better recoveries and coefficient of variances (CVs) were observed for PRiME HLB $\mu$ Elution (Figure 2).

The matrix effect of biological samples is mainly caused by endogenous components, such as carbohydrates, mineral salts, fats and other metabolites, especially phospholipids. Phospholipids structurally contain phosphate head groups with negative charges, while their quarternary amines confer a positive charge, which is known to cause significant LC-MS/MS matrix ionization effects [32,33]. Although applying matrix-matched calibration curves can partly compensate for signal suppression from the matrix effect, the sensitivity is also compromised. To compare the phospholipid clean-up efficiency of the HLB, PRiME HLB and PRiME HLB $\mu$ Elution techniques, characteristic ions with $m / z 184.0>184.0$ and $m / z 104.0>104.0$ formed from glycerophosphocholines (GPChos) and lysoglycerophosphocholines (Lyso-GPChos) in plasma extracts, respectively, were obtained after the clean-up procedures were monitored via in-source multiple reaction monitoring (IS MRM) [34]. Comparing the responses of extracts from the same pooled plasma, the PRiME-based method removed more than $90 \%$ of GPChos in the matrix, while PRiME HLB $\mu$ Elution showed the best adsorptivity for the selective clean-up of Lyso-GPChos (Figure 3).

Under all the optimized conditions, the pretreatment procedure in the PRiME HLB $\mu$ Elution method proved efficient. In our method, the matrix interference was well controlled and satisfactory recoveries were obtained. Furthermore, the preconditioning step was found unnecessary, and the 
evaporation step was replaced by simultaneous concentration with elution. Thus, the loss of analytes caused by evaporation was eliminated. As a result, the time required for the extraction and clean-up of 96 samples totaled approximately $15 \mathrm{~min}$, far quicker than the cartridge method. The reagent cost was also lower, and the volume of waste decreased from $2 \mathrm{~mL}$ to $600 \mu \mathrm{L}$ for each sample.

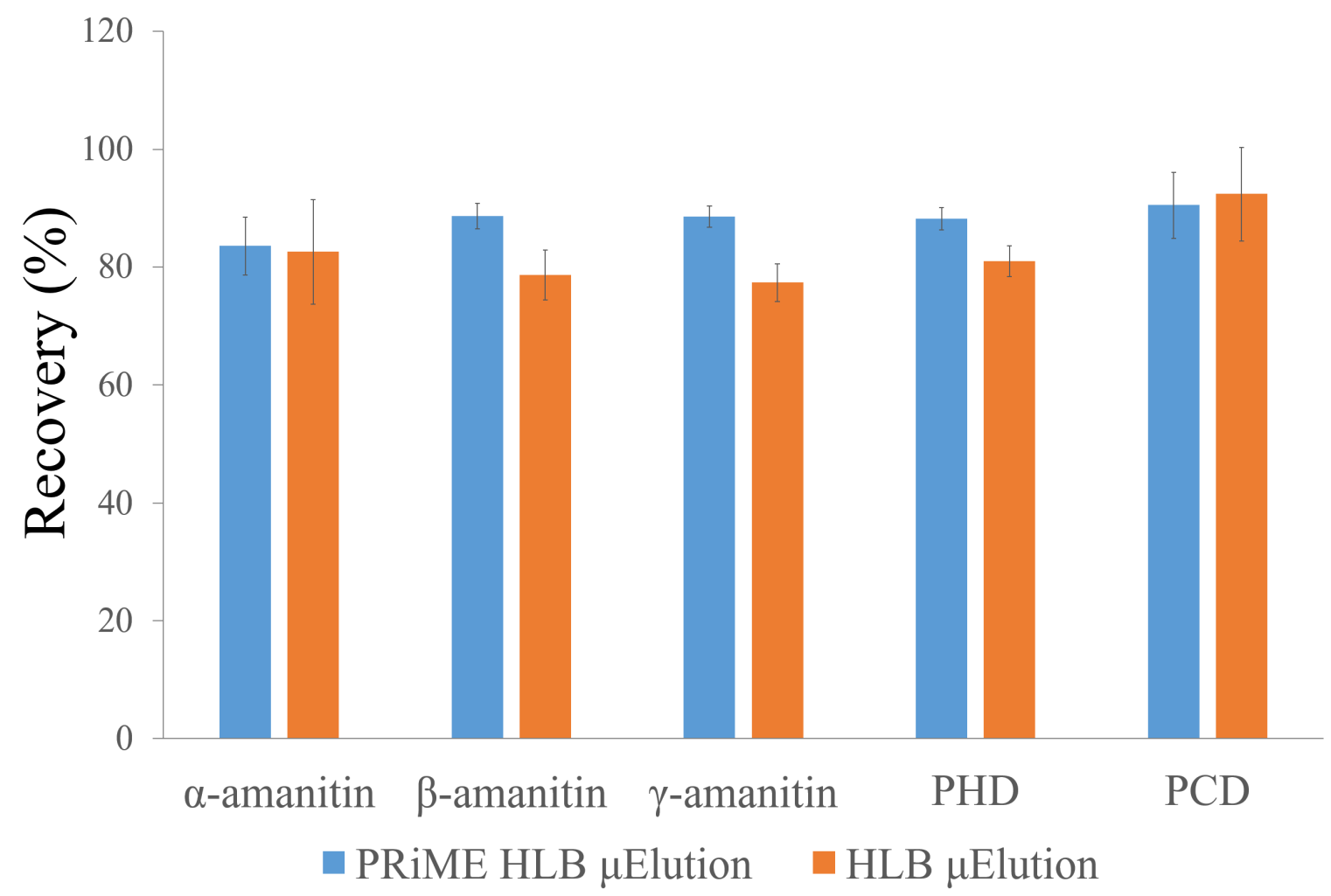

Figure 2. Comparison of extraction efficiency (recoveries \%) of HLB and PRiME HLB $\mu$ Elution for $10 \mathrm{ng} / \mathrm{mL} \alpha$-amanitin, $\beta$-amanitin, $\gamma$-amanitin, PHD and PCD in blank plasma-spiked sample $(n=3)$.

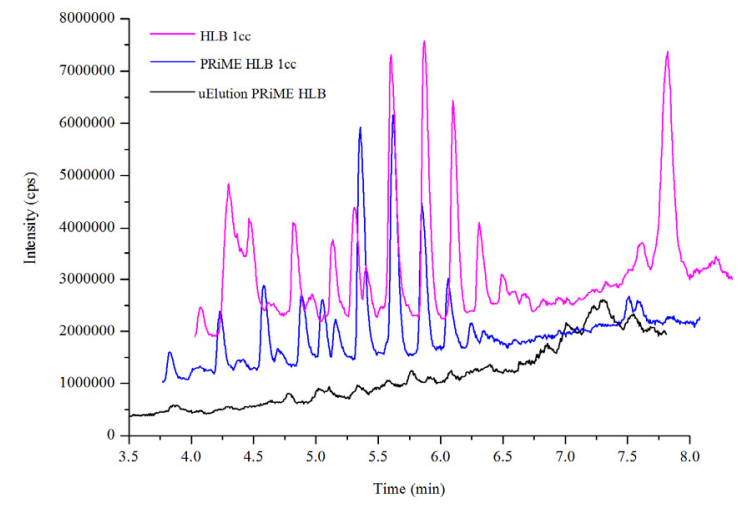

(a)

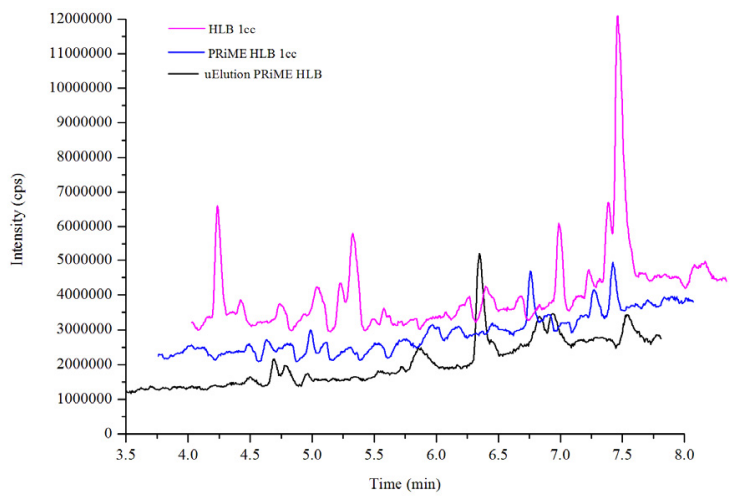

(b)

Figure 3. Comparison of LC/MS/MS chromatograms for IS-MRM transition for (a) GPChos, $m / z$ $184>$ 184; (b) Lyso-GPChos, m/z $104>104$ in plasma extracts, at $90 \mathrm{~V}$ cone voltage and $7 \mathrm{~V}$ collision energy.

\subsection{Method Validation}

Selectivity, accuracy, precision, matrix effects, linearity, and stability were validated for this method. 


\subsubsection{Specificity/Selectivity}

Twenty blank urine samples were taken from healthy volunteers and treated with the SPE procedure and LC-MS/MS method described in Materials and Methods section (section 3). The samples were then analyzed and compared with the corresponding spiked urine samples at the lower limit of quantification (LLOQ) level to check for possible interference with the detection of the analytes. Twenty samples of blank plasma and 20 samples of blank serum were equivalently analyzed for the same purpose. Representative chromatograms are shown in Figure 4. No obvious interfering peak from blank samples was detected.

\section{(a) plasma}
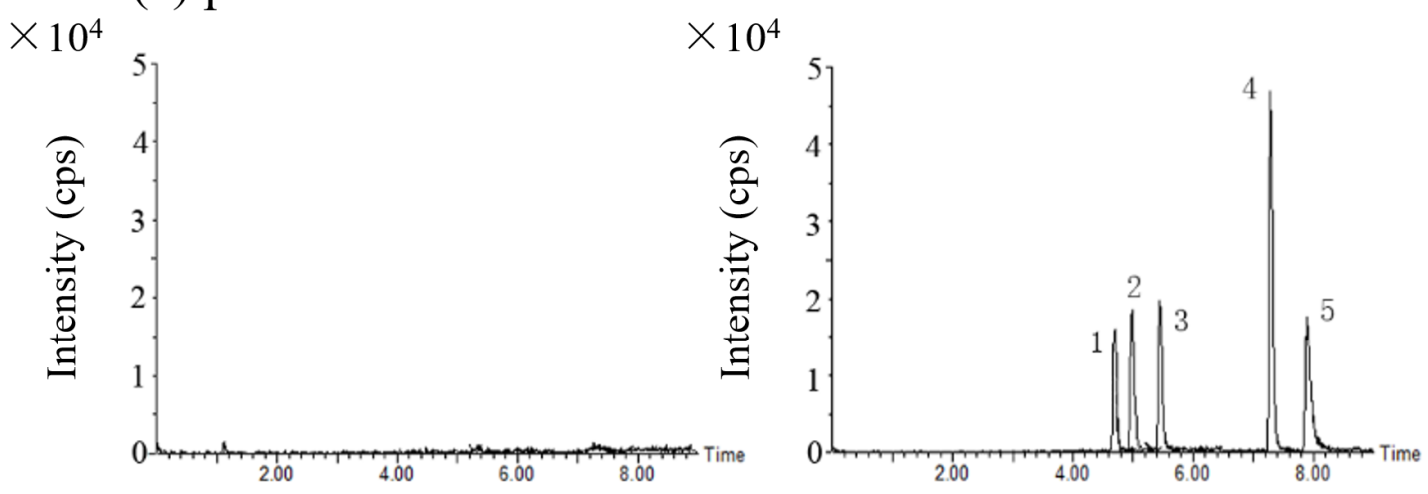

(b) serum
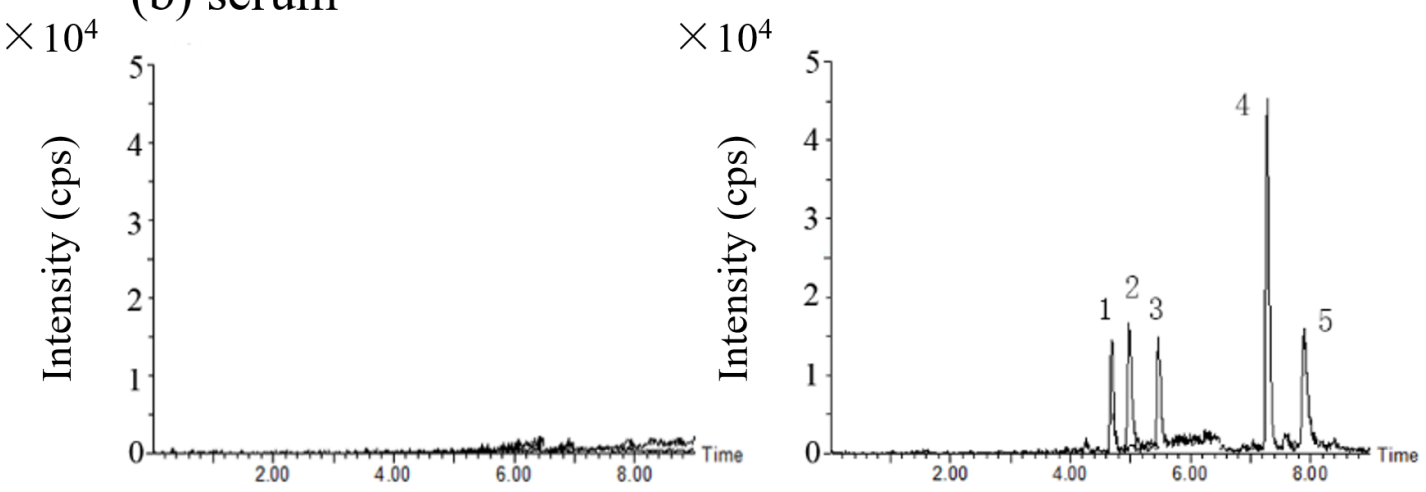

\section{(c) urine}
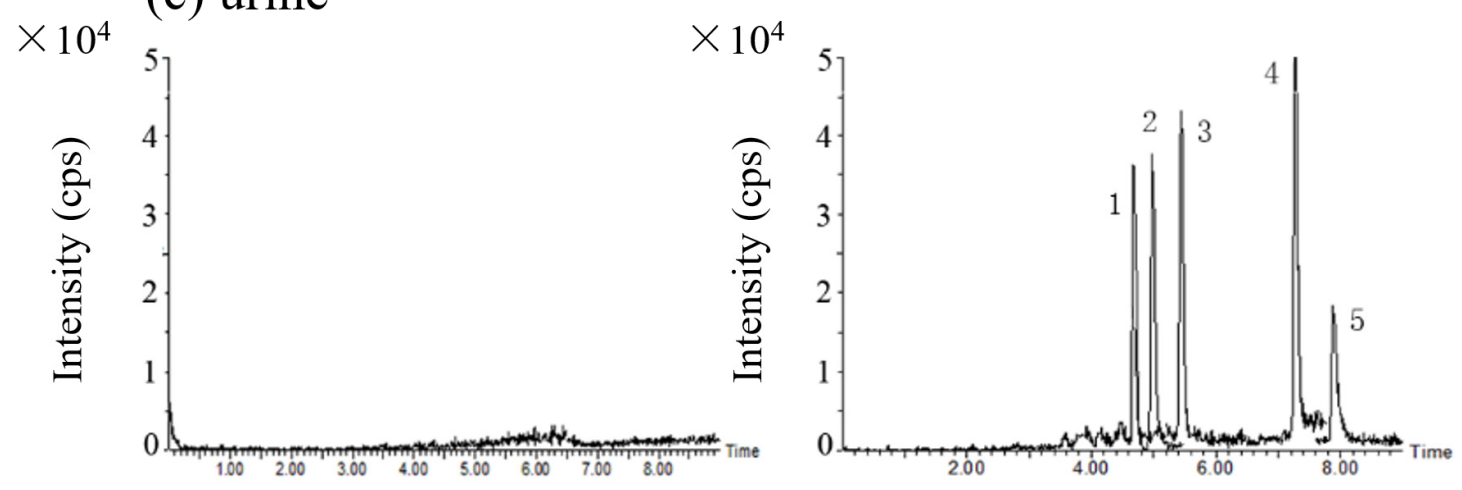

Figure 4. The LC-MS/MS MRM chromatograms of blank matrices (left) and (1) $\alpha$-amanitin, (2) $\beta$-amanitin, (3) $\gamma$-amanitin, (4) PHD and (5) PCD at their LLOQ levels in (a) plasma, (b) serum and (c) urine (right), respectively. The five quantification ion transitions $(\mathrm{m} / \mathrm{z} 919.5>86.0, \mathrm{~m} / \mathrm{z} 920.5>86.0$, $m / z 903.0>86.0, m / z 789.4>157.0, m / z 847.0>157.0$ ) are overlapped in the chromatograms. 


\subsubsection{Recovery, Matrix Effects, and Process Efficiency}

Three types of standard curves were set up to determine the absolute recoveries (RE), matrix effect (ME) and process efficiency (PE). Set I curves were standard solution curves, plotted using water-dissolved standard solutions of $0.2,0.5,1,2,5,10$ and $20 \mathrm{ng} / \mathrm{mL}$. The Set II curves were matrix-matched standard curves obtained by combining aliquots of working solutions followed by dilution with extracts of blank samples at concentrations of 1, 2, 5, 10, 20, 50 and $100 \mathrm{ng} / \mathrm{mL}$. They also served as calibration curves. Finally, the Set III curves were obtained from matrix-fortified standard samples, plotted by using extracts of blank pooled samples spiked before sample preparation at levels of $1,2,5,10,20,50$ and $100 \mathrm{ng} / \mathrm{mL}$ and serving as quality control (QC) samples. The ME, RE, and PE values were calculated with Matuszewski's algorithm [35]: ME was determined as the percentage ratio between the slope of Set II and the slope of Set I. RE was calculated as the percentage ratio of the slope of Set III to the slope of Set II. Finally, PE was obtained by multiplying the percentage ratio between the slopes of Set III and Set I by 100. In our research, the ME, representing the relative matrix effect [36], was studied to assess the interference from the sample matrix and the individual differences among biological fluids originating from different subjects or at various time points. We calculated the precision of the slopes of Set II standard lines, which were constructed for six different lots of urine, serum and plasma samples. The value of the matrix effect should be within $80 \%-120 \%$ and the CV of the relative matrix effects should be within 15\% [37]. RE, ME, and PE results with their CVs are shown in Table 2. The REs were $80.69 \%$ or more for amatoxins and $86.33 \%$ or more for phallotoxins. The intra-day precision ranged from $2.14 \%$ to $7.01 \%$, and the inter-day precision from $2.42 \%$ to $8.54 \%$. The signals of all analytes were matrix-enhanced or suppressed in various degrees and the CVs of the MEs calculated from the six lots of matrices were $6.80 \%$ or less.

Table 2. Recovery, intra-day and inter-day precision values for amatoxins and phallotoxins in human plasma, serum and urine.

\begin{tabular}{|c|c|c|c|c|c|c|c|c|}
\hline \multirow[t]{2}{*}{ Compound } & \multirow[t]{2}{*}{ Matrix } & \multirow{2}{*}{$\begin{array}{l}\text { Relative Matrix } \\
\text { Effect } \%(C V \%) \\
\qquad n=6\end{array}$} & \multirow{2}{*}{$\begin{array}{c}\text { Process } \\
\text { Efficiency } \\
\%\end{array}$} & \multirow[t]{2}{*}{$\begin{array}{l}\text { Recovery } \\
\% n=6\end{array}$} & \multicolumn{2}{|c|}{$\begin{array}{l}\text { Precision } \% \\
\quad n=6\end{array}$} & \multirow{2}{*}{$\begin{array}{c}\text { LOD } \\
\mathrm{ng} / \mathrm{g} \\
n=20\end{array}$} & \multirow{2}{*}{$\begin{array}{c}\text { LLOQ } \\
\text { ng/g } \\
n=20\end{array}$} \\
\hline & & & & & Intra-day & Inter-day & & \\
\hline \multirow{3}{*}{$\alpha$-amanitin } & plasma & 105.18 (3.05) & 87.84 & 83.51 & 5.86 & 6.68 & 0.5 & 1 \\
\hline & serum & $98.25(6.52)$ & 80.93 & 82.37 & 4.76 & 5.03 & 0.5 & 1 \\
\hline & urine & $91.92(6.80)$ & 77.43 & 84.24 & 3.94 & 4.02 & 1 & 2.5 \\
\hline \multirow{3}{*}{$\beta$-amanitin } & plasma & $103.37(4.44)$ & 91.56 & 88.58 & 5.43 & 2.42 & 0.5 & 1 \\
\hline & serum & $100.48(3.08)$ & 85.50 & 85.10 & 7.01 & 5.46 & 0.5 & 1 \\
\hline & urine & $96.80(3.85)$ & 78.11 & 80.69 & 3.68 & 3.22 & 0.5 & 1 \\
\hline \multirow{3}{*}{$\gamma$-amanitin } & plasma & $98.51(4.82)$ & 87.17 & 88.49 & 3.01 & 2.57 & 0.5 & 1 \\
\hline & serum & $105.47(3.97)$ & 95.11 & 90.18 & 2.14 & 4.04 & 1 & 2.5 \\
\hline & urine & $105.99(4.11)$ & 87.62 & 82.67 & 3.29 & 5.67 & 1 & 2.5 \\
\hline \multirow{3}{*}{ PHD } & plasma & $103.83(3.44)$ & 91.49 & 88.12 & 2.16 & 5.53 & 0.5 & 1 \\
\hline & serum & 103.57 (3.39) & 96.21 & 92.90 & 3.92 & 5.71 & 0.5 & 1 \\
\hline & urine & $103.84(3.36)$ & 91.01 & 87.65 & 3.44 & 4.03 & 0.5 & 1 \\
\hline \multirow{3}{*}{ PCD } & plasma & 99.03 (3.17) & 99.46 & 90.44 & 6.21 & 8.54 & 0.5 & 1 \\
\hline & serum & $105.77(2.54)$ & 91.79 & 86.78 & 2.72 & 3.04 & 0.5 & 1 \\
\hline & urine & $94.58(3.62)$ & 81.65 & 86.33 & 4.09 & 7.22 & 0.5 & 1 \\
\hline
\end{tabular}

\subsubsection{Linearity/Work Range}

The matrix-matched calibration curves, i.e., the Set II standard curves, were prepared as mentioned above, over a linear range from the LLOQ of each analyte at seven concentrations between 0 and $100 \mathrm{ng} / \mathrm{mL}$. The $\alpha-, \beta-, \gamma$-Amanitin, PHD and PCD spiked in blank sample extracts at concentrations of $1,2,5,10,25,50,75$ and $100 \mathrm{ng} / \mathrm{mL}$ were analyzed. One calibration curve was analyzed each day for three days. Linearity was evaluated using the least squares regression algorithm with a weighting factor of 1 /concentration. Correlation coefficients were obtained and residual analysis was performed 
to test the linear model. All calibration curves gave good linearities for plasma, serum and urine, with correlation coefficient $\left(r^{2}\right)$ values greater than 0.998 (Table 3).

Table 3. The slopes of regression equations and correlation coefficients of target compounds in a different matrix.

\begin{tabular}{|c|c|c|c|c|}
\hline Compound & $\begin{array}{l}\text { Slopes of Set I (CV\%), } \\
\text { Correlation Coefficient } \\
\qquad\left(r^{2}\right)(n=6)\end{array}$ & Matrix & $\begin{array}{l}\text { Slopes of Set II }(\mathrm{CV} \%), \\
\text { Correlation Coefficient } \\
\qquad\left(r^{2}\right)(n=6)\end{array}$ & $\begin{array}{l}\text { Slopes of Set III }(\mathrm{CV} \%) \text {, } \\
\text { Correlation Coefficient } \\
\qquad\left(r^{2}\right)(n=6)\end{array}$ \\
\hline \multirow{3}{*}{$\alpha$-amanitin } & \multirow{3}{*}{$1306.37(0.84), 0.9993$} & plasma & $1374.01(3.05), 0.9996$ & $1243.57(5.86), 0.9998$ \\
\hline & & serum & $1294.35(6.52), 0.9993$ & $1011.68(4.76), 0.9989$ \\
\hline & & urine & $1200.82(6.80), 0.9998$ & $1061.60(3.94), 0.9998$ \\
\hline \multirow{3}{*}{$\beta$-amanitin } & \multirow{3}{*}{1534.87 (1.03), 0.9998} & plasma & $1510.67(4.44), 9.9998$ & 1338.17 (2.43), 0.9994 \\
\hline & & serum & $1542.29(3.08), 0.9983$ & $1306.25(6.01), 0.9978$ \\
\hline & & urine & $1485.85(3.85), 0.9987$ & $1235.27(2.68), 0.9995$ \\
\hline \multirow{3}{*}{$\gamma$-amanitin } & \multirow{3}{*}{$952.75(0.96), 0.9996$} & plasma & $938.58(4.82), 0.9998$ & $933.87(2.01), 0.9992$ \\
\hline & & serum & $1004.89(3.97), 0.9986$ & $875.13(2.14), 0.9967$ \\
\hline & & urine & $1009.82(4.11), 0.9999$ & 906.19 (3.29), 0.9998 \\
\hline \multirow{3}{*}{ PHD } & \multirow{3}{*}{$3434.45(0.95), 0.9998$} & plasma & $3595.76(3.44), 0.9998$ & $3600.11(2.16), 0.9976$ \\
\hline & & serum & 3557.01 (3.39), 0.9997 & $3125.76(3.92), 0.9994$ \\
\hline & & urine & $3566.29(3.36), 0.9998$ & $3304.45(3.44), 0.9993$ \\
\hline \multirow{3}{*}{ PCD } & \multirow{3}{*}{$1442.27(0.99), 0.9998$} & plasma & $1428.30(3.17), 0.9999$ & $1434.52(6.21), 0.9992$ \\
\hline & & serum & $1525.57(2.54), 0.9997$ & $1177.65(2.72), 0.9987$ \\
\hline & & urine & 1364.07 (3.62), 0.9998 & 1339.23 (4.09), 0.9983 \\
\hline
\end{tabular}

\subsubsection{Limit of Detection and Lower Limit of Quantification}

The limit of detection (LOD) and LLOQ were obtained from the intensity of 20 blank pooled urine, serum and plasma samples, respectively (Table 3). The LOD was determined as signal:noise ratio $>3: 1$ for the chromatographic response. The LLOQ was determined as signal:noise ratio > 10:1. The LOD and LLOQ obtained in the present study are at similar or lower levels compared with previous LC-MS/MS reports, but cover five compounds including both amatoxins and phallotoxins. Previous research into the kinetics of amatoxin poisoning in humans has been limited, while the kinetic data for phallotoxins remain unknown. According to Jaeger [9], plasma amatoxins were detected at 8-90 ng/mL and 23.5-162 ng/mL for $\alpha$ - and $\beta$-amanitin, respectively. In urine, amatoxin concentrations were 48-4820 ng/mL and 75-7103 ng/mL for $\alpha$ - and $\beta$-amanitin, respectively. Therefore, the present method is sufficiently sensitive to detect amanitins in urine and blood in cases of mushroom poisoning in humans.

\subsubsection{Stability}

The stability of the analytes in the studied matrices was evaluated using the Set III samples (blank matrix spiked with analytes before extraction at levels of 1, 2, 5, 10, 20, 50, 75 and $100 \mathrm{ng} / \mathrm{mL}$ ) at different storage time periods (after one, two, four and eight weeks). The results of the stability evaluation are shown in Table 4 . The recoveries $(75.15 \%-100.86 \%)$ of the analytes in plasma, serum and urine showed no significant changes as a function of storage time, with precisions ranging from $1.98 \%$ to $11.99 \%$. 
Table 4. Stability data for amatoxins and phallotoxins in human plasma, serum and urine $(n=3)$.

\begin{tabular}{lccccc}
\hline \multirow{2}{*}{ Compound } & Matrix & One Week & Two Weeks & Four Weeks & Eight Weeks \\
\cline { 3 - 6 } & & \multicolumn{4}{c}{ Recovery\% (Precision\%) } \\
\hline \multirow{3}{*}{$\alpha$-amanitin } & plasma & $87.12(5.52)$ & $90.95(3.52)$ & $94.56(7.23)$ & $93.73(7.31)$ \\
& serum & $83.31(4.57)$ & $87.25(8.74)$ & $85.15(6.79)$ & $84.42(10.04)$ \\
& urine & $81.03(3.25)$ & $86.32(5.54)$ & $83.64(4.54)$ & $80.43(3.40)$ \\
\hline \multirow{5}{*}{$\beta$-amanitin } & plasma & $82.56(5.72)$ & $80.77(4.72)$ & $84.78(8.79)$ & $83.38(9.09)$ \\
& serum & $75.15(2.98)$ & $83.26(5.08)$ & $80.63(5.08)$ & $87.55(4.99)$ \\
& urine & $87.54(3.76)$ & $83.94(3.60)$ & $85.42(2.65)$ & $89.12(2.95)$ \\
\hline \multirow{5}{*}{-amanitin } & plasma & $91.33(1.98)$ & $96.68(11.99)$ & $94.44(3.90)$ & $96.61(8.30)$ \\
& serum & $85.27(2.88)$ & $88.71(4.58)$ & $86.04(6.82)$ & $83.14(3.82)$ \\
& urine & $78.34(3.25$ & $83.42(6.94)$ & $85.23(5.43)$ & $76.23(5.43)$ \\
\hline \multirow{2}{*}{ PHD } & plasma & $95.87(3.12)$ & $98.66(3.22)$ & $97.42(6.26)$ & $93.40(6.27)$ \\
& serum & $90.03(7.38)$ & $97.04(3.28)$ & $95.11(5.24)$ & $100.86(6.72)$ \\
& urine & $80.62(6.10)$ & $80.62(5.05)$ & $85.24(9.05)$ & $90.50(7.55)$ \\
\hline \multirow{2}{*}{ PCD } & plasma & $96.41(4.34)$ & $90.65(3.36)$ & $92.18(2.35)$ & $88.35(4.56)$ \\
& serum & $85.24(3.66)$ & $83.49(5.16)$ & $82.55(4.36)$ & $87.52(6.01)$ \\
& urine & $87.55(5.45)$ & $84.56(7.91)$ & $86.49(6.88)$ & $83.92(3.85)$ \\
\hline
\end{tabular}

\section{Materials and Methods}

\subsection{Reagents and Materials}

The $\alpha-, \beta-, \gamma$-Amanitin and PHD ( $\geqslant 90 \%$ purity) were purchased from Enzo Life Sciences (Farmingdale, NY, USA); PCD ( $\geqslant 85 \%$ purity) was purchased from Sigma-Aldrich (St. Louis, MO, USA). Ultra-pure water was obtained from Millipore System (Molsheim, France). Ammonium acetate and formic acid were obtained from Merck (Darmstadt, Germany); acetonitrile and methanol were obtained, respectively, from JT Baker (Deventer, Holland) and Fisher Chemical (Leicestershire, UK). Oasis HLB 1 cc (30 mg), Oasis WAX 1 cc (30 mg), Oasis WCX 1 cc (30 mg), and Oasis HLB 1 cc (30 mg) cartridges, as well as Oasis HLB $\mu$ Elution and Oasis PRiME HLB $\mu$ Elution plates, were purchased from Waters (Milford, MA, USA); ACQUITY UPLC HSS T3 $(2.1 \mathrm{~mm} \times 100 \mathrm{~mm}, 1.8 \mu \mathrm{m})$, ACQUITY UPLC BEH C18 $(2.1 \mathrm{~mm} \times 100 \mathrm{~mm}, 1.7 \mu \mathrm{m})$ and CORTECS UPLC C18+ $(2.1 \mathrm{~mm} \times 100 \mathrm{~mm}, 1.6 \mu \mathrm{m})$ separation columns were purchased from Waters (Milford, MA, USA).

Human blank plasma, serum, and urine samples were obtained from healthy volunteers. The authentic biological samples were collected from 31 patients of suspected mushroom poisoning in the People's Hospital of Chuxiong Yi Autonomous Prefecture. All patients gave their informed consent for inclusion before they participated in the study. The study was conducted in accordance with the Declaration of Helsinki, and the protocol was approved by the Ethics Committee of The 307th Hospital of Chinese People's Liberation Army (Project identification code 20140354181939) in 25 February 2014.

\subsection{Preparation of Calibration Standards}

Individual stock standard solutions of $\alpha$-amanitin, $\beta$-amanitin, $\gamma$-amanitin, PHD and PCD were prepared in methanol $(1.0 \mathrm{mg} / \mathrm{mL})$ and stored in the dark at $-20^{\circ} \mathrm{C}$. Standard working solutions of each analyte ranging from $100 \mathrm{ng} / \mathrm{mL}$ to $1 \mu \mathrm{g} / \mathrm{L}$ were prepared by serial dilution of the stock solutions with ultra-pure water and stored at $4{ }^{\circ} \mathrm{C}$.

Three types of standard curve were prepared in our research. Set I curves were standard solution curves, plotted by using water-dissolved standard solutions of $0.2,0.5,1,2,5,10,15$ and $20 \mathrm{ng} / \mathrm{mL}$. The Set II curves were matrix-matched standard curves obtained by combining aliquots of working solutions followed by dilution with extracts of blank samples at concentrations of 1, 2, 5, 10, 20, 50, 75 and $100 \mathrm{ng} / \mathrm{mL}$, which also served as calibration curves. Finally, the Set III curves were obtained from 
matrix-fortified standard samples, plotted by using extracts of blank pooled samples spiked before sample preparation at levels of 1,2,5, 10, 20, 50, 75 and $100 \mathrm{ng} / \mathrm{mL}$ and serving as QC samples.

\subsection{Sample Preparation}

\subsubsection{Urine Samples}

The mixture of $100 \mu \mathrm{L}$ urine sample and $100 \mu \mathrm{L}$ ammonium acetate buffer (1M, pH 5) was slowly loaded onto the PRiME HLB $\mu$ Elution 96-well plate. Each well was washed with $200 \mu \mathrm{L}$ of $5 \%$ methanol in water $(v / v)$ twice. The compounds were eluted with $25 \mu \mathrm{L}$ of $95 \%$ methanol in water $(v / v)$ twice, collected in a clean 96-well collection plate, and subsequently diluted with $450 \mu \mathrm{L}$ of mobile phase solution $(0.2 \%$ formic acid in distilled water) prior to LC-MS/MS analysis.

\subsubsection{Plasma and Serum Samples}

The mixture of $100 \mu \mathrm{L}$ plasma or serum sample and $100 \mu \mathrm{L}$ of $4 \% \mathrm{H}_{3} \mathrm{PO}_{4}$ in distilled water was slowly loaded onto the PRiME HLB $\mu$ Elution 96-well plate. Then each well was washed with $200 \mu \mathrm{L}$ of $5 \%$ methanol in water twice. The retained compounds were eluted into a clean 96-well collection plate with $25 \mu \mathrm{L} 95 \%$ methanol in water twice. The eluent was simply diluted with $450 \mu \mathrm{L}$ of the mobile phase solution before LC-MS/MS analysis.

\subsection{LC-MS/MS Analysis}

The analytes were identified and quantified on a xevo TQ MS detector (Waters, Milford, MA, USA) coupled to the UPLC ACQUILITY system (Waters, Milford, MA, USA). In the chromatographic system, a CORTEX UPLC C18+ $(2.1 \mathrm{~mm} \times 100 \mathrm{~mm}, 1.6 \mu \mathrm{m})$ column was used for separation. The mobile phases were $0.2 \%$ formic acid in water (mobile phase $\mathrm{A}$ ) and $0.2 \%$ formic acid in methanol (mobile phase B). A gradient program was started with $95 \% \mathrm{~A}$ and $5 \% \mathrm{~B}$, with phase B increasing linearly to $30 \%$ in the first $4 \mathrm{~min}$, linearly to $40 \%$ at $6 \mathrm{~min}$ and remaining constant for $2 \mathrm{~min}$. The mobile phase then returned to the initial composition and equilibrated for $2 \mathrm{~min}$ prior to the next injection. The total run time for each sample was $10 \mathrm{~min}$. The flow rate was $0.2 \mathrm{~mL} / \mathrm{min}$, and the injection volume was $5 \mu \mathrm{L}$. The column temperature was $60^{\circ} \mathrm{C}$.

MS/MS detection was carried out on a Waters xevo TQ-S mass spectrometer. MRM was used to measure the target analytes with the positive electrospray ionization (ESI+) mode. The MS conditions were as follows: capillary voltage, $3.0 \mathrm{kV}$; desolvation gas $\left(600 \mathrm{~L} / \mathrm{h}, 500{ }^{\circ} \mathrm{C}\right)$, nitrogen; collision gas $(0.15 \mathrm{~mL} / \mathrm{min})$, argon. The MRM parameters for each compound and ion transition were optimized, as summarized in Table 1.

\section{Method Application}

Authentic samples from 31 patients with acute accidental poisoning with wild mushrooms were collected from the hospital at 48-192 h post-ingestion, mostly after $72 \mathrm{~h}$, including 24 urine samples, 30 plasma samples and three serum samples. They were analyzed using the present method. The $\alpha$-Amanitin and PCD were found in two samples of urine. For one sample, $\alpha$-amanitin and PCD were at 6.02 and $2.24 \mathrm{ng} / \mathrm{mL}$ respectively, and for the other, they were at 1.98 and $2.52 \mathrm{ng} / \mathrm{mL}$, respectively (the chromatograms are shown in Figure 5). However, none of the target toxins were detected in plasma or serum. Detailed urine sample information is given in the Supplementary Materials. This result is consistent with previous studies showing that amanitins can be eliminated very rapidly from blood within $30 \mathrm{~h}$ after ingestion, but can remain for three days in urine [9]. Besides, only a few of the wild mushrooms the patients consumed were obtained and identified as the genus Amanita. The other possible explanation for the low positive rate is that the species of the wild mushrooms in most cases is unknown, and they probably are not amatoxin- or phallotoxin-producing species, but they may also cause gastrointestinal (GI) symptoms and increased AST or ALT levels, such as the genus Russula and Boletus do. In the positive cases, the patients presented with early GI 
symptoms and elevated liver enzymes. The ingested mushrooms were also collected and identified morphologically and phylogenetically as Amanita exitialis, a poisonous mushroom that has only been reported in China, and contains $\alpha, \beta$-amanitin and PCD among the five target toxins [38].

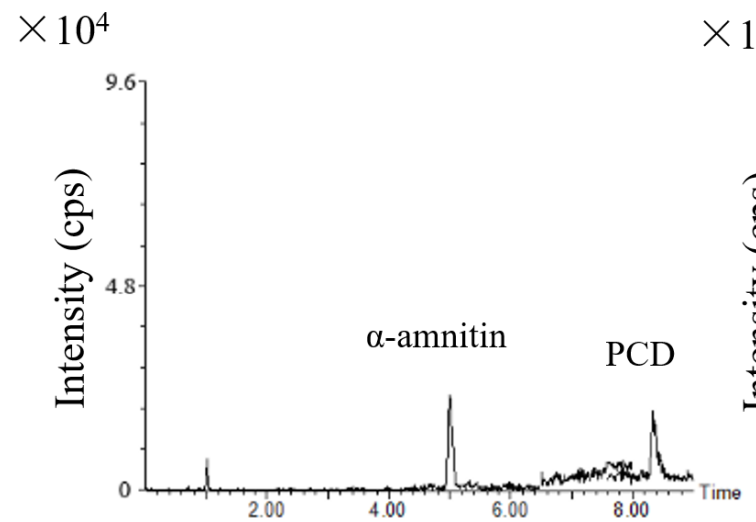

(a)

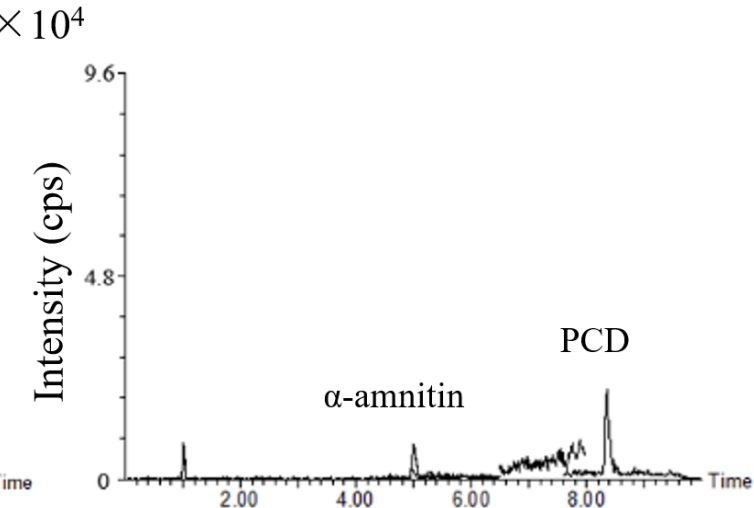

(b)

Figure 5. The overlapped LC-MS/MS MRM chromatograms of positive urine samples (a) $\alpha$-amanitin at $6.02 \mathrm{ng} / \mathrm{mL}$ and PCD at $2.24 \mathrm{ng} / \mathrm{mL}$; (b) $\alpha$-amanitin at $1.98 \mathrm{ng} / \mathrm{mL}$ and PCD at $2.52 \mathrm{ng} / \mathrm{mL}$.

\section{Conclusions}

A rapid, convenient and high-throughput LC-MS/MS method was developed for the simultaneous analysis of $\alpha$-amanitin, $\beta$-amanitin, $\gamma$-amanitin, PHD and PCD in urine, plasma and serum using the PRiME $\mu$ Elution technique for labor-reducing and time-saving purposes. The method has been fully validated, and was successfully applied to authentic biological fluid sample analysis. This method is faster, more convenient and more cost-effective than existing methods; it also avoids internal standards and expensive instrumentation. In summary, this method is suitable for the routine emergency toxicological and clinical determination of amatoxins and phallotoxins in human biological fluids.

Supplementary Materials: The following are available online at www.mdpi.com/2072-6651/8/5/128/s1. Figure S1: The proposed fragmentation of the analytes by MS/MS mode to their products, Table S1: The information of 24 urine samples with mushroom poisoning.

Acknowledgments: This work was financially supported by Chinese National Scientific Research Special-Purpose Project in Public Health Profession (No. 2015SQ00192), Young Scholar Scientific Research Fund of China National Center for Food Safety Risk Assessment (No. 2015008) and National Nature Science Foundation of China Youth Science Foudation (31501814).

Author Contributions: Shuo Zhang, Yunfeng Zhao and Chengye Sun conceived and designed the experiments; Shuo Zhang performed the experiments; Shuo Zhang, Dawei Chen analyzed the data; Haijiao Li, Yizhe Zhang and Qunmei Yao contributed to sampling; Shuo Zhang and Shuang Zhou drafted the manuscript.

Conflicts of Interest: The authors declare no conflict of interest.

\section{References}

1. Chen, Z.H.; Zhang, P.; Zhang, Z.G. Investigation and analysis of 102 mushroom poisoning casesin Southern China from 1994 to 2012. Fungal Divers. 2014, 64, 123-131. [CrossRef]

2. Wang, R.; Gao, Y.J.; Ding, F.; Xie, R.H.; Wang, X.Y.; Li, Q. Epidemiological analysis on mushroom poisoning in China 2004-2011. Chin. J. Public Health 2014, 30, 158-161.

3. Wieland, T. The toxic peptides from Amanita mushrooms. Int. J. Peptideprotein Res. 1983, 22, 257-276. [CrossRef]

4. Luo, H.; Hallen-Adams, H.E.; Scott-Craig, J.S.; Walton, J.D. Ribosomal biosynthesis of $\alpha$-amanitin in Galerina marginata. Fungal Genet. Biol. 2012, 49, 123-129. [CrossRef] [PubMed]

5. Mas, A. Mushrooms, amatoxins and the liver. J. Hepatol. 2005, 42, 166-169. [CrossRef] [PubMed] 
6. Vargas, N.; Bernal, A.; Sarria, V.; Franco-Molano, A.; Restrepo, S. Amatoxin and phallotoxin composition in species of the genus Amanitain Colombia: A taxonomic perspective. Toxicon 2011, 58, 583-590. [CrossRef] [PubMed]

7. Sgambelluri, R.M.; Epis, S.; Sassera, D.; Luo, H.; Angelos, E.R.; Walton, J.D. Profiling of amatoxins and phallotoxins in the genus lepiota by liquid chromatography combined with UV absorbance and mass spectrometry. Toxins 2014, 6, 2336-2347. [CrossRef] [PubMed]

8. Enjalbert, F.; Rapior, S.; Nouguier-Soulé, J.; Guillon, S.; Amouroux, N.; Cabot, C. Treatment of amatoxin poisoning: 20-year retrospective analysis. J. Toxicol. 2002, 40, 715-757. [CrossRef]

9. Jaeger, A.; Jehl, F.; Flesch, F.; Sauder, P.; Kopferschmitt, J. Kinetics of amatoxins in human poisoning: Therapeutic implications. J. Clin. Toxicol. 1993, 31, 63-80. [CrossRef]

10. Rentsch, K.M. Laboratory diagnostics in acute poisoning: Critical overview. Clin. Chem. Lab. Med. 2010, 48, 1381-1387. [CrossRef] [PubMed]

11. Faulstich, H.; Zobeley, S.; Trischmann, H. A rapid radioimmunoassay, using a nylon support, for amatoxins from Amanita mushrooms. Toxicon 1982, 20, 913-924. [CrossRef]

12. Faulstich, H.; Trischmann, H.; Zobeley, S. A radioimmunoassay for amanitin. FEBS Lett. 1975, 56, 312-315. [CrossRef]

13. Homann, J.; Rawer, P.; Bleyl, H.; Matthes, K.; Heinrich, D. Early detection of amatoxins in human mushroom poisoning. Arch. Toxicol. 1986, 59, 190-191. [CrossRef] [PubMed]

14. Abuknesha, R.A.; Maragkou, A. A highly sensitive and specific enzyme immunoassay for detection of beta-amanitin in biological fluids. Anal. Bioanal. Chem. 1993, 379, 853-860.

15. Butera, R.; Locatelli, C.; Coccini, T.; Manzo, L. Diagnostic accuracy of urinary amanitin in suspected mushroom poisoning: A pilot study. J. Toxicol. Clin. Toxicol. 2004, 42, 901-912. [CrossRef] [PubMed]

16. Bruggemann, O.; Meder, M.; Freitag, R. Analysis of amatoxins $\alpha$-amanitin and $\beta$-amanitin in toadstool extracts and body fluids by capillary zone electrophoresis with photodiode array detection. J. Chromatogr. A 1996, 744, 167-176. [CrossRef]

17. Robinson-Fuentes, V.; Jaimes-Sánchez, J.; García-Aguilar, L.; Gómez-Peralta, M.; Vázquez-Garciduenas, M.; Vázquez-Marrfo, G. Determination of $\alpha$ - and $\beta$-amanitin in clinical urine samples by Capillary Zone Electrophoresis. J. Pharm. Biomed. Anal. 2008, 47, 913-917. [CrossRef] [PubMed]

18. Rieck, W.; Platt, D. High-performance liquid chromatographic method for the determination of $\alpha$-amanitin and phalloidin in human plasma using the column-switching technique and its application in suspected cases of poisoning by the green species of amanita mushroom (Amanita phalloides). J. Chromatogr. 1988, 425, 121-134. [PubMed]

19. Zhou, Z.; Cao, M.; Zhou, L.; Zuo, X.; Tang, Y. Determination of $\alpha$-amanitin in human serum by solid-phase extraction coupled with HPLC-UV. LC GC N. Am. 2011, 29, 672-677.

20. Tagliaro, F.; Schiavon, G.; Bontempelli, G.; Carli, G.; Marigo, M. Improved high-performance liquid chromatographic determination with amperometric detection of $\alpha$-amanitin in human plasma based on its voltammetric study. J. Chromatogr. 1991, 563, 299-311. [CrossRef]

21. Defendenti, C.; Bonacina, E.; Mauroni, M.; Gelosa, L. Validation of a high performance liquid chromatographic method for alpha amanitin determination in urine. Forensic Sci. Int. 1998, 92, 59-68. [CrossRef]

22. Garcia, J.; Costa, V.M.; Baptista, P.; Bastos, M.L.; Carvalho, F. Quantification of alpha-amanitin in biological samples by HPLC using simultaneous UV-diode array and electrochemical detection. J. Chromatogr. B 2015, 997, 85-95. [CrossRef] [PubMed]

23. Leite, M.; Freitas, A.; Azul, A.M.; Barbosa, J.; Costa, S.; Ramos, F. Development, optimization and application of an analytical methodology by ultra performance liquid chromatography-Tandem mass spectrometry for determination of amanitins in urine and liver samples. Anal. Chim. Acta 2013, 799, 77-87. [CrossRef] [PubMed]

24. Maurer, H.H.; Schmitt, C.J.; Weber, A.A.; Kraemer, T. Validated electrospray liquid chromatographic-mass spectrometric assay for the determination of the mushroom toxins $\alpha$ - and $\beta$-amanitin in urine after immunoaffinity extraction. J. Chromatogr. B 2000, 748, 125-135. [CrossRef]

25. Chung, W.; Tso, S.; Sze, S. Separation of polar mushroom toxins by mixed-mode hydrophilic and ionicInteraction liquid chromatography-Electrospray ionization-mass spectrometry. J. Chromatogr. Sci. 2007, 45, 104-111. [CrossRef] [PubMed] 
26. Tanahashi, M.; Kaneko, R.; Hirata, Y.; Hamajima, M.; Arinobu, T.; Ogawa, T.; Ishii, A. Simple analysis of $\alpha$-amanitin and $\beta$-amanitin in human plasma by liquid chromatography-mass spectrometry. Forensic Toxicol. 2010, 28, 110-114. [CrossRef]

27. Nomura, M.; Suzuki, Y.; Kaneko, R.; Ogawa, T.; Hattori, H.; Seno, H.; Ishii, A. Simple and rapid analysis of amatoxins using UPLC-MS-MS. Forensic Toxicol. 2012, 30, 185-192. [CrossRef]

28. Helfer, A.G.; Meyer, M.R.; Michely, J.A.; Maurer, H.H. Direct analysis of the mushroom poisons $\alpha$ - and $\beta$-amanitin in human urine using a novel on-line turbulent flow chromatography modecoupled to liquid chromatography-high resolution-massspectrometry/mass spectrometry. J. Chromatogr. A 2014, 1325, 92-98. [CrossRef] [PubMed]

29. Tomkova, J.; Ondra, P.; Válka, I. Simultaneous determination of mushroom toxins $\alpha$-amanitin, $\beta$-amanitin and muscarine in human urine by solid-phase extraction and ultra-high-performance liquid chromatography coupled with ultra-high-resolution TOF mass spectrometry. Forensic Sci. Int. 2015, 251, 209-213. [CrossRef] [PubMed]

30. Gonmori, K.; Minakata, K.; Suzuki, M.; Yamagishi, I.; Nozawa, H.; Hasegawa, K.; Wurita, A.; Watanabe, K.; Suzuki, O. MALDI-TOF mass spectrometric analysis of $\alpha$-amanitin, $\beta$-amanitin, and phalloidin in urine. Forensic Toxicol. 2012, 30, 179-184. [CrossRef]

31. Vetter, J. Toxins of Amanita phalloides. Toxicon 1997, 36, 13-24. [CrossRef]

32. Chambers, E.; Wagrowski-Diehl, D.M.; Lu, Z.; Mazzeo, J.R. Systematic and comprehensive strategy for reducing matrix effects in LC/MS/MS analyses. J. Chromatogr. B 2007, 852, 22-34. [CrossRef] [PubMed]

33. Carmical, J.; Brown, S. The impact of phospholipids and phospholipid removal on bioanalytical method performance. Biomed. Chromatogr. 2016. [CrossRef] [PubMed]

34. Little, J.L.; Wempe, M.F.; Buchanan, C.M. Liquid chromatography-mass spectrometry/mass spectrometry method development for drug metabolism studies: Examining lipid matrix ionization effects in plasma. J. Chromatogr. B 2006, 833, 219-230. [CrossRef] [PubMed]

35. Matuszewski, B.K.; Constanzer, M.L.; Chavez-Eng, C.M. Strategies for the assessment of matrix effect in quantitative bioanalytical methods based on HPLC-MS/MS. Anal. Chem. 2003, 75, 3019-3030. [CrossRef] [PubMed]

36. Matuszewski, B.K. Standard line slopes as a measure of a relative matrix effect in quantitative HPLC-MS bioanalysis. J. Chromatogr. B 2006, 830, 293-300. [CrossRef] [PubMed]

37. Guideline on bioanalytical method validation. European Medicines Agency. Available online: http://www. ema.europa.eu/docs/en_GB/document_library/Scientific_guideline/2011/08/WC500109686.pdf (accessed on 12 February 2016).

38. Deng, Q.W.; Li, T.H.; Xi, P.G.; Gan, L.X.; Xiao, Z.D.; Jiang, Z.D. Peptide toxin components of Amanita exitialis basidiocarps. Mycologia 2011, 103, 946-949. [CrossRef] [PubMed] 